\title{
In Vitro Screening of Three Commercial Cannabis-Based Products on ATP-Binding Cassette and Solute-Carrier Transporter Function
}

\author{
Lyndsey L. Anderson, ${ }^{1-3, \dagger}$ Maia G. Etchart, ${ }^{1,2, \dagger}$ Laura MacNair, ${ }^{4}$ M. Hunter Land, ${ }^{4}$ Irina A. Mosesova, ${ }^{4}$
} Marcel O. Bonn-Miller, ${ }^{4, \dagger}$ and Jonathon C. Arnold ${ }^{1-3, \dagger, *}$

\begin{abstract}
Introduction: Legalization of medicinal cannabis around the world has led to an increase in the use of commercial cannabis-based products in the community. These cannabis-based products are being used in combination with conventional drugs to treat a variety of health conditions. Moreover, recreational cannabis-based products may be used in combination with other drugs. In this setting, there is increased potential for drug-drug interactions (DDIs) involving commercial cannabis-based products. Since DDIs can lead to serious adverse events, drug regulatory bodies require that every investigational drug be evaluated for DDI potential at metabolic enzymes and transporters. However, this seldom occurs for cannabis-based products due to legislation in many jurisdictions allowing a direct pathway to market. This study aimed to examine the inhibitory potential of three commercially available cannabis-based products at human ATP-binding cassette (ABC) and solute-carrier (SLC) transporters.

Materials and Methods: Three commercial cannabis-based products (Spectrum Yellow ${ }^{\mathrm{TM}}$, Tweed Argyle, and Spectrum Red ${ }^{\mathrm{TM}}$ ) that contain differing concentrations of cannabidiol (CBD) and $\Delta^{9}$-tetrahydrocannabinol $\left(\Delta^{9}-\mathrm{THC}\right)$ were evaluated for DDI potential at 12 drug transporters. HEK293 cells or vesicles expressing human $A B C$ transporters (ABCB1, $A B C C 2, A B C G 2$, or $A B C B 11)$ and SLC transporters (SLC22A1, SLC22A2, SLC22A6, SLC22A8, SLCO1B1, SLCO1B3, SLC47A1, and SLC47A2) were used to measure transporter function.

Results: Spectrum Yellow and Tweed Argyle inhibited ABCG2 transporter function. The $I C_{50}$ value of Spectrum Yellow based on CBD and $\Delta^{9}$-THC content was $4.5 \mu \mathrm{M}$ for CBD and $0.20 \mu \mathrm{M}$ for $\Delta^{9}-\mathrm{THC}$, and the $\mathrm{IC}_{50}$ value of Tweed Argyle was $9.3 \mu \mathrm{M}$ for CBD and $6.0 \mu \mathrm{M}$ for $\Delta^{9}$-THC. Tweed Argyle also inhibited ABCB11 transporter function with an IC 50 value of $11.9 \mu \mathrm{M}$ for CBD and $7.7 \mu \mathrm{M}$ for $\Delta^{9}$-THC. SLC22A6, SLC22A1, SLC22A2, SLCO1B1, and SLCO1B3 transporter functions were modestly inhibited by high concentrations of the cannabis-based products. The three cannabis-based products did not inhibit ABCB1, ABCC2, SLC47A1, SLC47A2, or SLC22A8 transporters. Discussion: Novel findings were that the cannabis-based products inhibited ABCB11, SLC22A6, SLC22A1, SLC22A2, SLCO1B1, and SLCO1B3 (although modestly in most instances). Spectrum Yellow and Tweed Argyle potently inhibited ABCG2, and future in vivo DDI studies could be conducted to assess whether cannabis products affect the pharmacokinetics of medications that are ABCG2 substrates.
\end{abstract}

Keywords: ABC transporters; cannabis; cannabis-based products; SLC transporters

\footnotetext{
'Brain and Mind Centre, The University of Sydney, Sydney, Australia.

${ }^{2}$ Lambert Initiative for Cannabinoid Therapeutics, The University of Sydney, Sydney, Australia.

${ }^{3}$ Discipline of Pharmacology, Faculty of Medicine and Health, The University of Sydney, Sydney, Australia.

${ }^{4}$ Canopy Growth Corporation, Smiths Falls, Ontario, Canada.

†These four authors contributed equally.
}

*Address correspondence to: Jonathon C. Arnold, PhD, Brain and Mind Centre, The University of Sydney, Building F, Level 6, 94 Mallett Street, Camperdown, NSW 2050, Australia, E-mail: jonathon.arnold@sydney.edu.au 


\section{Introduction}

The widespread legalization of medicinal and recreational cannabis use worldwide has led to a proliferation of cannabis-based products becoming available on the market. With commercial cannabis-based products increasingly being used in the community for both medicinal and recreational purposes, there is an increased potential for a drug-drug interaction (DDI) between cannabis-based products and conventional treatments.

When considering DDIs, the initial focus tends to be on drug metabolism and cytochrome P450 (CYP) enzymes. The CYP450 superfamily of proteins metabolize $\sim 75 \%$ of medically important drugs and competition between two drugs for the same metabolizing enzyme can lead to serious DDIs. ${ }^{1}$ Both $\Delta^{9}$-tetrahydrocannabinol $\left(\Delta^{9}\right.$-THC) and cannabidiol (CBD), the major phytocannabinoids in cannabis, have been shown to be potent inhibitors of CYP450s. ${ }^{1}$ Indeed, a clinically significant DDI mediated by CYP450s occurs between Epidiolex ${ }^{\circledR}$, a purified preparation of $\mathrm{CBD}$, and clobazam, a first-line treatment for intractable childhood epilepsies. ${ }^{2,3}$

However, another important source of DDIs occurs at transporters. Drug transporters facilitate the movement of substrate molecules across biological membranes. These transporters regulate the accumulation of substrates in various organ tissues such as brain, gut, liver, and kidney; therefore, a DDI at transporters can profoundly affect drug bioavailability and disposition, thereby altering concentrations within organ tissues. ATP-binding cassette $(\mathrm{ABC})$ and solute-carrier (SLC) transporters are the most extensively characterized families of transporters.

The ABC transporter superfamily includes P-glycoprotein (ABCB1), breast cancer resistance protein (ABCG2), bile salt export protein (ABCB11), and multidrug resistance-associated proteins (ABCC1 and ABCC2). ${ }^{4,5}$ Studies have shown that both CBD and $\Delta^{9}$-THC inhibit $\mathrm{ABCB} 1, \mathrm{ABCG} 2$, and $\mathrm{ABCC} 1$; however, the effects of $\mathrm{CBD}$ and $\Delta^{9}$-THC on other ABC transporters are unknown. ${ }^{6-10}$

Moreover, there have been no studies examining the effects of CBD and $\Delta^{9}$-THC on the SLC superfamily of transporters relevant to DDIs. Notably, CBD inhibits SLC29A1/ENT1, which is predicted to contribute to its therapeutic effects. ${ }^{11}$ SLC transporters that will be studied here include organic cation transporters (SLC22A1 and SLC22A2), organic anion transporting polypeptides (SLCO1B1 and SLCO1B3), organic anion transporters (OATs) (SLC22A6 and SLC22A8), and human multidrug and toxin extrusion transporters (SLC47A1 and SLC47A2).

Because DDIs can lead to serious adverse events, drug regulatory agencies mandate the evaluation of the DDI potential of every new investigational drug at transporters and metabolic enzymes. However, this seldom occurs for cannabis-based products due to legislation in many jurisdictions allowing a direct pathway to market without the need for formal drug development practices. This study sought to examine the inhibitory potential of three commercial cannabis products at human drug transporters, including various members in the ABC and SLC superfamilies. The three cannabis-based products contain differing concentrations of $\mathrm{CBD}$ and $\Delta^{9}$-THC, and are available on the market as either medicinal (Spectrum Yellow ${ }^{\mathrm{TM}}$ and Spectrum Red ${ }^{\mathrm{TM}}$ ) or recreational (Tweed Argyle) products.

\section{Materials and Methods}

Cannabis-based products

Spectrum Yellow, Tweed Argyle, and Spectrum Red are three commercially available cannabis-based products that are delivered orally (Canopy Growth Corporation, Inc., Toronto, Canada). CBD and $\Delta^{9}$-THC content in these products were provided as follows: Spectrum Yellow, $0.84 \mathrm{mg} / \mathrm{mL}(2.7 \mathrm{mM}) \Delta^{9}-\mathrm{THC}$ and $19 \mathrm{mg} / \mathrm{mL}$ (60.4 mM) CBD in medium-chain triglyceride (MCT) oil; Tweed Argyle, $5.2 \mathrm{mg} / \mathrm{mL}(16.5 \mathrm{mM}) \Delta^{9}$-THC and $8 \mathrm{mg} / \mathrm{mL}$ (25.4 mM) CBD in sesame oil; Spectrum Red, $25 \mathrm{mg} / \mathrm{mL}(80 \mathrm{mM}) \Delta^{9}$-THC and $<0.5 \mathrm{mg} / \mathrm{mL}$ $(<1.5 \mathrm{mM}) \mathrm{CBD}$ in MCT oil. Concentrations of additional cannabinoids and terpenes are presented in Supplementary Table S1. Concentrations of flavonoids were not examined. All cannabis-based products were stored at room temperature in sealed amber glass bottles and used within 2 months from opening.

Spectrum Yellow and Spectrum Red are being used to treat a variety of health conditions. Dosing is variable and typically titrated by the prescribing health care professional based on patient response.

\section{Rationale for cannabis-based product} concentrations tested

Peak plasma $\Delta^{9}$-THC concentrations measured after oral administration of $10-15 \mathrm{mg}$ Marinol $^{\circledR}$ (synthetic $\Delta^{9}$-THC) are $16.9 \mathrm{ng} / \mathrm{mL}(0.054 \mu \mathrm{M}) .{ }^{12}$ Spectrum Yellow, which contains the lowest $\Delta^{9}$-THC content, was diluted, so that the $\Delta^{9}$-THC concentration tested $(0.046 \mu \mathrm{M})$ was consistent with plasma $\Delta^{9}$-THC 
concentrations achieved with Marinol treatment allowing for relevant translation of our findings. The same dilution factor $(\sim 58,695)$ was used for Tweed Argyle and a Spectrum Red stock (previously diluted 1:10). Following European Medicines Agency (EMA) guidelines, concentrations 50 -fold higher than the low dose were also tested. Final $\Delta^{9}$-THC concentrations tested were 0.046 and $2.3 \mu \mathrm{M}$ (Spectrum Yellow), 0.28 and $14 \mu \mathrm{M}$ (Tweed Argyle), and 0.14 and $7 \mu \mathrm{M}$ (Spectrum Red).

\section{Chemicals}

Decynium-22, 1-methyl-4-phenylpyridinium iodide (MPP), metformin, cimetidine, estrone 3-sulfate (ES), $p$-aminohippuric acid (PAH), probenecid, elacridar, rifampicin, cyclosporineA, taurocholate, benzobromarone, 5(6)-carboxy-2', $7^{\prime}$-dichlorofluorescein (CDCF), and rhodamine123 were purchased from Sigma-Aldrich (Steinheim, Germany). Sulfobromophthalein was purchased from Carl Roth (Karlsruhe, Germany). Radiolabeled substrates, ${ }^{3} \mathrm{H}$-MPP, ${ }^{14} \mathrm{C}$-metformin, ${ }^{3} \mathrm{H}$-ES, ${ }^{3} \mathrm{H}-\mathrm{PAH}$, and ${ }^{3} \mathrm{H}$-taurocholate, were purchased from American Radiolabeled Chemicals (St. Louis). ${ }^{3} \mathrm{H}$ sulfobromophthalein was purchased from Hartmann Analytics (Brunswick, Germany). ${ }^{3} \mathrm{H}$-taurocholate was purchased from PerkinElmer (Waltham, MA).

\section{Cell culture}

HEK293 cells were used for all transporter studies and grown in high glucose Dulbecco's Modified Eagle Medium supplemented with $10 \%$ fetal bovine serum, $100 \mathrm{U} / \mathrm{mL}$ penicillin, and $100 \mu \mathrm{g} / \mathrm{mL}$ streptomycin in a humidified $5 \% \mathrm{CO}_{2}$ atmosphere at $37^{\circ} \mathrm{C}$. Cells stably expressing human SLC22A1, SLC22A2, SLC22A6,
SLC22A8, SLCO1B1, SLCO1B3, SLC47A1, SLC47A2, $A B C B 1, A B C C 2, A B C G 2$, or ABCB11 were generated by PortaCellTec Biosciences GmbH (Göttingen, Germany), and all experiments were conducted by them. Control cells were transfected with empty vector pcDNA5.

\section{Uptake and efflux transport assays}

Cells $(200,000 /$ well $)$ were plated into 24-well, poly-Dlysine $(0.1 \mathrm{mg} / \mathrm{mL})$ plates and cultured for 3 days. In cells expressing $\mathrm{ABCB} 1$, media were supplemented with tetracycline $(1 \mu \mathrm{g} / \mathrm{mL})$ and butyrate $(5 \mathrm{mM})$ to induce expression. Media were aspirated, and each well was rinsed with $0.5 \mathrm{~mL}$ incubation buffer (HBSS supplemented with $20 \mathrm{mM}$ HEPES, pH 7.4).

For uptake assays, cells were incubated for $20 \mathrm{~min}$ at $37^{\circ} \mathrm{C}$, then the incubation buffer was replaced with $200 \mu \mathrm{L}$ reaction mixture, consisting of substrate (radiolabeled and nonradiolabeled) and inhibitor (cannabisbased products or control inhibitors) in incubation buffer and incubated at $37^{\circ} \mathrm{C}$. Details regarding experimental conditions for each transporter are provided in Table 1 . Uptake was terminated by aspirating the reaction mixture and rinsing each well with ice-cold phosphate-buffered saline (PBS, $0.4 \mathrm{~mL}$ ). Cells were solubilized in $1 \mathrm{~N} \mathrm{NaOH}(0.6 \mathrm{~mL})$ overnight and transferred to scintillation vials with $2.5 \mathrm{~mL}$ scintillation solvent (Roti ${ }^{\circledR}$ ECOplus; Carl Roth). A Tri-Carb 2810 Scintillation Counter (PerkinElmer) was used to measure ${ }^{3} \mathrm{H}$ and ${ }^{14} \mathrm{C}$ content.

For efflux assays, cells were incubated for $60 \mathrm{~min}$ at $37^{\circ} \mathrm{C}$ with $200 \mu \mathrm{L}$ incubation buffer containing

Table 1. Experimental Conditions for Transporter Assays

\begin{tabular}{|c|c|c|c|c|c|c|c|}
\hline Transporter & $\begin{array}{c}\text { Common } \\
\text { names }\end{array}$ & Assay type & Substrate & $\begin{array}{c}\text { Substrate } \\
\text { concentration, } \mu \mathrm{M}\end{array}$ & Inhibitor & $\begin{array}{c}\text { Inhibitor } \\
\text { concentration, } \mu \mathrm{M}\end{array}$ & $\begin{array}{l}\text { Incubation } \\
\text { time, min }\end{array}$ \\
\hline $\mathrm{ABCB} 1$ & MDR1, P-gp & Efflux & Rhodamine123 & 10 & Cyclosporine A & 10 & 30 \\
\hline $\mathrm{ABCC} 2$ & MRP2 & Efflux & CDCF & 5 & Benzobromarone & 100 & 10 \\
\hline$A B C G 2$ & BCRP & $\begin{array}{l}\text { Vesicle-based } \\
\text { uptake }\end{array}$ & ES $\left({ }^{3} \mathrm{H}-\mathrm{ES}\right)$ & $1(0.01)$ & Elacridar & 5 & 1 \\
\hline ABCB11 & BSEP & $\begin{array}{l}\text { Vesicle-based } \\
\text { uptake }\end{array}$ & $\begin{array}{l}\text { Taurocholate } \\
\left({ }^{3} \mathrm{H} \text {-taurocholate }\right)\end{array}$ & $1(0.02)$ & Cyclosporine & 50 & 2 \\
\hline SLC22A1 & OCT1 & Uptake & MPP $\left({ }^{3} \mathrm{H}-\mathrm{MPP}\right)$ & $10(0.002)$ & Decynium-22 & 50 & 1 \\
\hline SLC22A2 & OCT2 & Uptake & MPP $\left({ }^{3} \mathrm{H}-\mathrm{MPP}\right)$ & $10(0.002)$ & Decynium-22 & 50 & 1 \\
\hline SLC22A6 & OAT1 & Uptake & PAH ( $\left.{ }^{3} \mathrm{H}-\mathrm{PAH}\right)$ & $10(0.1)$ & Probenecid & 100 & 5 \\
\hline SLC22A8 & OAT3 & Uptake & ES $\left({ }^{3} \mathrm{H}-\mathrm{ES}\right)$ & $1(0.01)$ & Probenecid & 100 & 1 \\
\hline SLCO1B1 & OATP1B1 & Uptake & ES $\left({ }^{3} \mathrm{H}-\mathrm{ES}\right)$ & $0.03(0.01)$ & Rifampicin & 50 & 1 \\
\hline SLCO1B3 & OATP1B3 & Uptake & BSP $\left({ }^{3} \mathrm{H}-\mathrm{BSP}\right)$ & $0.05(0.02)$ & Rifampicin & 5 & 7 \\
\hline SLC47A1 & MATE1 & Uptake & Metformin $\left({ }^{14} \mathrm{C}\right.$-metformin) & $20(1)$ & Cimetidine & 50 & 1 \\
\hline SLC47A2 & MATE2K & Uptake & Metformin $\left({ }^{14} \mathrm{C}\right.$-metformin) & $90(1)$ & Cimetidine & 50 & 1 \\
\hline
\end{tabular}

Radiolabeled substrates and concentrations shown in parentheses.

ABC, ATP-binding cassette; ES, estrone 3-sulfate; MPP, 1-methyl-4-phenylpyridinium iodide; PAH, p-aminohippuric acid; SLC, solute carrier; MATE, multidrug and toxin extrusion; OAT, organic anion transporter; OATP, organic anion transporting polypeptide; CDCF, 5(6)-carboxy-2' $7^{\prime}$-dichlorofluorescein; BSP, sulfobromophthalein. 
substrate (Table 1). Cells were rinsed, then incubation buffer $(0.5 \mathrm{~mL})$ containing inhibitor (cannabis-based products or control inhibitors) was added and incubated at $37^{\circ} \mathrm{C}$. Supernatants were transferred to a clean plate, and a TECAN Ultra plate reader (Tecan Group Ltd., Männedorf, Switzerland) was used to take fluorescence readings (excitation $490 \mathrm{~nm}$, emission $535 \mathrm{~nm}$ ).

Drugs were tested with a dimethyl sulfoxide concentration $\leq 1 \%$. Cellular protein amounts were determined from a parallel plate through Bradford assay. A Wallac Victor2 plate reader (PerkinElmer) was used to measure absorption $(595 \mathrm{~nm})$. Quantification of protein was performed by comparing samples to standards with known amounts of protein.

\section{Vesicle-based uptake assays}

Inside-out membrane vesicles were prepared from cells expressing empty vector, ABCG2 or ABCB $11 .{ }^{13}$ Reaction mixtures containing substrate (radiolabeled and nonradiolabeled), inhibitor (cannabis-based products or control inhibitor), and ATP were warmed to $37^{\circ} \mathrm{C}$, and uptake was initiated by the addition of the membrane suspension and incubated at $37^{\circ} \mathrm{C}$ (Table 1 ). Reactions were terminated by the addition of ice-cold PBS $(1 \mathrm{~mL})$, then filtered through a glass fiber filter under vacuum. Filters were rinsed with ice-cold PBS $(3 \mathrm{~mL})$, dried and transferred to scintillation vials containing $1 \mathrm{~N} \mathrm{NaOH}(0.6 \mathrm{~mL})$ and $3 \mathrm{~mL}$ scintillation solvent to measure ${ }^{3} \mathrm{H}$ content.

\section{Data analysis}

For influx transporter assays, initial uptake velocities $(v, \mathrm{pmol} / \mathrm{mg}$ protein/min) were calculated for each transporter using the following equations:

$$
\begin{gathered}
v=\frac{\mathrm{RA}}{\mathrm{SA} \times P \times t} \\
\mathrm{SA}=\frac{\mathrm{St}}{[s] \times V \times 10^{6}}
\end{gathered}
$$

Radioactivity of experimental sample (RA, dpm), incubation interval $(t, \mathrm{~min})$, and protein quantity $(P, \mathrm{mg}$ protein) were used. Substrate radioactivity (SA, $\mathrm{dpm} / \mathrm{pmol}$ ) was calculated from St (dpm, radioactivity of a standard aliquot), substrate concentration $([\mathrm{S}], \mu \mathrm{M})$, and volume $(V, L)$. Concentrationresponse curves were generated for ABCG2 and $\mathrm{ABCB} 11$ since $\geq 50 \%$ inhibition was observed in the initial screen. Data were fit to a three-parameter logistic equation in Graphpad PRISM 7 (San Diego) to determine $\mathrm{IC}_{50}$ values.

For efflux transporter assays, initial efflux velocities ( $v, \mathrm{pmol} / \mathrm{mg}$ protein $/ \mathrm{min}$ ) were calculated for each transporter using the following equation:

$$
v=\frac{\mathrm{FL} \times D \times V}{P \times S \times t} .
$$

Fluorescence of experimental sample (FL), dilution $(D, 1 / 5)$, volume $(V, L)$, protein quantity $(P, \mathrm{mg}$ protein), slope of calibration curve $\left(S, 1 / \mu \mathrm{M} \times 10^{6}\right)$, and incubation interval $(t, \mathrm{~min})$ were used.

Statistical comparisons were made across treatments using one-way analysis of variance followed by Dunnett's post hoc from Graphpad PRISM, and $p<0.05$ was considered statistically significant.

\section{Results and Discussion}

Cannabis-based products do not inhibit multidrug resistance proteins

Multidrug resistance proteins are $\mathrm{ABC}$ transporters that function to efflux endogenous and xenobiotic substrates across biological membranes. We examined the inhibitory potential of the three commercial cannabisbased products on substrate efflux in cells expressing ABCB1 or ABCC2. ABCB1-mediated efflux of rhodamine123 was significantly increased in the presence of these products; however, efflux was increased to the same extent in control cells suggesting a nonspecific effect (Fig. 1A, B). The cannabis-based products did not affect ABCC2-mediated efflux of CDCF (Fig. 1C, D).

ABCB1, also commonly known as P-glycoprotein, has numerous drug substrates, including analgesics, antipsychotics, anticonvulsants, anticancer, antibiotics, and antiarrhythmic drugs, ${ }^{14}$ and in vivo transportermediated DDIs are likely to arise from interactions at ABCB1. Here, each cannabis-based product surprisingly increased efflux of rhodamine123, although this appeared to be nonspecific since a similar effect was observed in control cells. A nonspecific effect could be the result of the cannabis-based products disrupting cellular membrane integrity; however, this is unlikely because under the same incubation conditions, the ABCC2 substrate CDCF did not exhibit increased efflux. One explanation is that the cannabis-based products induced greater ABCB1 expression, as CBD and $\Delta^{9}$-THC increase expression of ABCB1. ${ }^{15,16}$ In human 


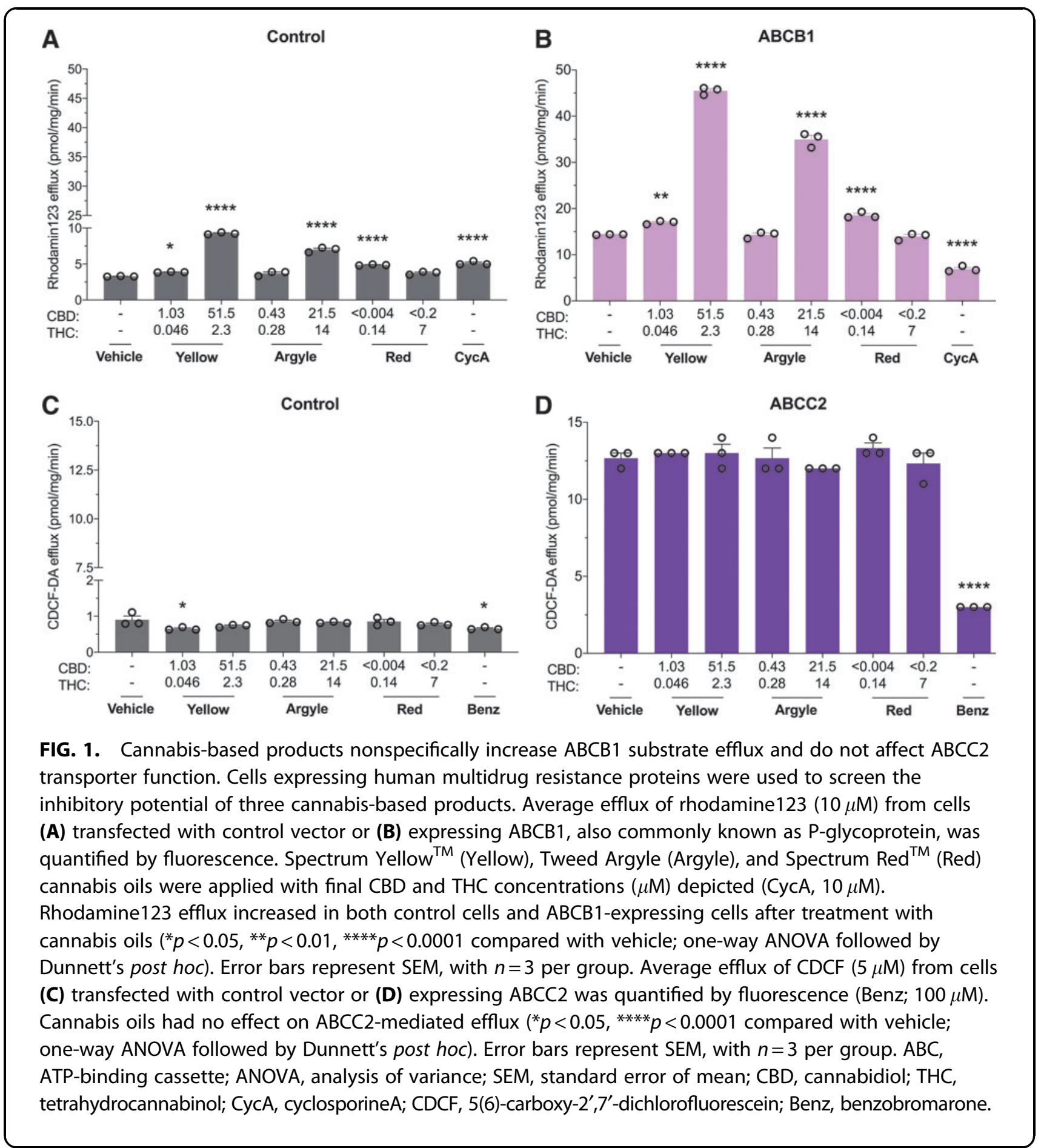

acute lymphoblastic leukemia cells, both $\Delta^{9}$-THC and CBD increased ABCB1 mRNA expression associated with increased rhodamine123 accumulation after a $4 \mathrm{~h}$ incubation period. ${ }^{15}$ While shorter incubation times were not measured in that study, it is unlikely that increased ABCB1 mRNA expression would occur in the $30 \mathrm{~min}$ incubation period used here. The nonspecific effects of the cannabis products and cyclosporinA in the control cells might imply weak disruption of endogenously expressed efflux transporters in HEK293 
cells, as cyclosporin $\mathrm{A}$ is not a selective $\mathrm{ABCB} 1$ transport inhibitor and rhodamine 123 is not a specific substrate for $\mathrm{ABCB} 1 .{ }^{17,18}$ In any case, further research is needed to determine the cause of the increased rhodamine123 efflux associated with the cannabis-based products, although it appears to be independent of ABCB1.

Prior studies have reported that $\mathrm{CBD}$ inhibits ABCB1-mediated efflux of rhodamine123. ${ }^{6,19}$ However, in a different study phytocannabinoids CBD, $\Delta^{9}$-THC, or cannabinol (CBN) did not inhibit ABCB1-mediated rhodamine123 but promoted a subtle but significant increase in rhodamine123 efflux. ${ }^{20}$ Considering that a study reported that phytocannabinoids CBD, CBN, and cannabispirol decreased ABCB1-mediated rhodamine123 efflux but cannabidiolic acid, $\Delta^{9}$-THC, and $\Delta^{9}$-tetrahydrocannabinolic acid increased its efflux, the phytocannabinoids in the cannabis-based products likely exert complex and potentially competing effects on $\mathrm{ABCB} 1$ transporter function. $^{19}$

Spectrum Yellow and Tweed Argyle inhibit breast cancer resistance protein

ABCG2 is another ABC efflux transporter. High concentrations of Spectrum Yellow and Tweed Argyle significantly inhibited ABCG2-mediated uptake of ${ }^{3} \mathrm{H}$-ES (76\%, $p<0.0001$ and 89\%, $p<0.0001$, respectively). Inhibition of ABCG2 was further characterized by generating concentration-response curves. The $\mathrm{IC}_{50}$ value of Spectrum Yellow was $4.5 \mu \mathrm{M}$ for CBD and $0.20 \mu \mathrm{M}$ for $\Delta^{9}$-THC (Fig. 2C). The $\mathrm{IC}_{50}$ value of Tweed Argyle was $9.3 \mu \mathrm{M}$ for CBD and $6.0 \mu \mathrm{M}$ for $\Delta^{9}$-THC (Fig. 2D).

The $\mathrm{IC}_{50}$ values of Spectrum Yellow and Tweed Argyle had similar CBD concentrations (4.5 and $9.3 \mu \mathrm{M})$ irrespective of $\Delta^{9}$-THC concentrations $(0.20$ and $6.0 \mu \mathrm{M}$ ), suggesting CBD might mediate the inhibitory effect. Moreover, the $\Delta^{9}$-THC-dominant Spectrum Red product displayed very modest (27\%) inhibitory effects on ABCG2 function and only at the lower concentration tested. CBD-mediated inhibition of ABCG2 is consistent with an ex vivo human placental perfusion assay showing CBD increased placental barrier permeability to glyburide. ${ }^{8}$ Whereas inhibition of ABCG2 by these cannabis-based products seemed to be independent of $\Delta^{9}$-THC, previous in vitro studies have shown that $\Delta^{9}$-THC inhibited mouse and human ABCG2 function with a similar potency to $\mathrm{CBD} .{ }^{9}$ One final point that merits consideration is that these products are plant extracts containing a multitude of other phytochemicals including terpenes. It remains possible that these components might also contribute to the inhibitory effects of these products on ABCG2 function.

Since ABCG2 mediates the efflux of numerous drugs such as antimicrobials, anti-cancer agents, rheumatoid arthritis drugs and cholesterol-lowering statins, any impact on transporter function could translate into a clinically meaningful DDI. ${ }^{21,22}$ The United States Food and Drug Administration (FDA) guidelines state that orally administered drugs should be further examined in vivo when $\mathrm{I}_{\text {gut }} / \mathrm{IC}_{50} \geq 10\left(\mathrm{I}_{\text {gut }}=\right.$ dose of inhibitor $/ 250 \mathrm{~mL}$ ). Based on this equation if doses of Spectrum Yellow and Tweed Argyle being used in the community result in CBD doses $>3.5$ and $7.3 \mathrm{mg}$, then in vivo DDI studies would normally be recommended according to conventional drug development practices. Definitive conclusions on whether these in vitro results have any clinical significance can only be drawn after the completion of a human DDI study.

Tweed Argyle inhibited bile salt export pump

Bile salt export pump (ABCB11) transporters are essential for transporting bile acid from the liver into the bile. ${ }^{23}$ High-dose Tweed Argyle significantly inhibited ABCB11-mediated uptake of ${ }^{3} \mathrm{H}$-taurocholate (59\%, $p=0.0003$ ) (Fig. 3B). The inhibition of ABCB11 was further characterized by generating a concentration-response curve. The $\mathrm{IC}_{50}$ value of Tweed Argyle were $11.9 \mu \mathrm{M} \mathrm{CBD}$ and $7.7 \mu \mathrm{M} \Delta^{9}$-THC (Fig. 3C). Conversely, Spectrum Red increased ABCB11-mediated uptake of substrate ( $p=0.0001$ and $p=0.0028)$ (Fig. 3B).

Dysfunction of the ABCB11 transporter through loss-of-function mutations or drug inhibition can lead to cholestasis and liver injury. ${ }^{23}$ Consequently, the International Transporter Consortium encourages that investigational new drugs be evaluated for ABCB11 inhibition. ${ }^{24}$ Our data show that Tweed Argyle, a cannabis-based product, inhibits $\mathrm{ABCB} 11$ function. While current FDA guidelines for evaluating DDI potential of investigational new drugs do not include ABCB11, the EMA recommends that drugs should be examined in vivo when the $\mathrm{IC}_{50} \leq 50 \times C_{\max }$ (unbound). Based on this an in vivo DDI study would be recommended for Tweed Argyle if plasma concentrations of CBD and $\Delta^{9}$-THC achieved were $>2.38 \mu \mathrm{M}(748 \mathrm{ng} / \mathrm{mL})$ and $1.54 \mu \mathrm{M}(484 \mathrm{ng} / \mathrm{mL})$, respectively. Unfortunately, no human pharmacokinetic data are available for these three cannabis-based products, so it is difficult to predict with certainty whether the concentrations above could be attained after the administration of Tweed Argyle. On the one hand, it 

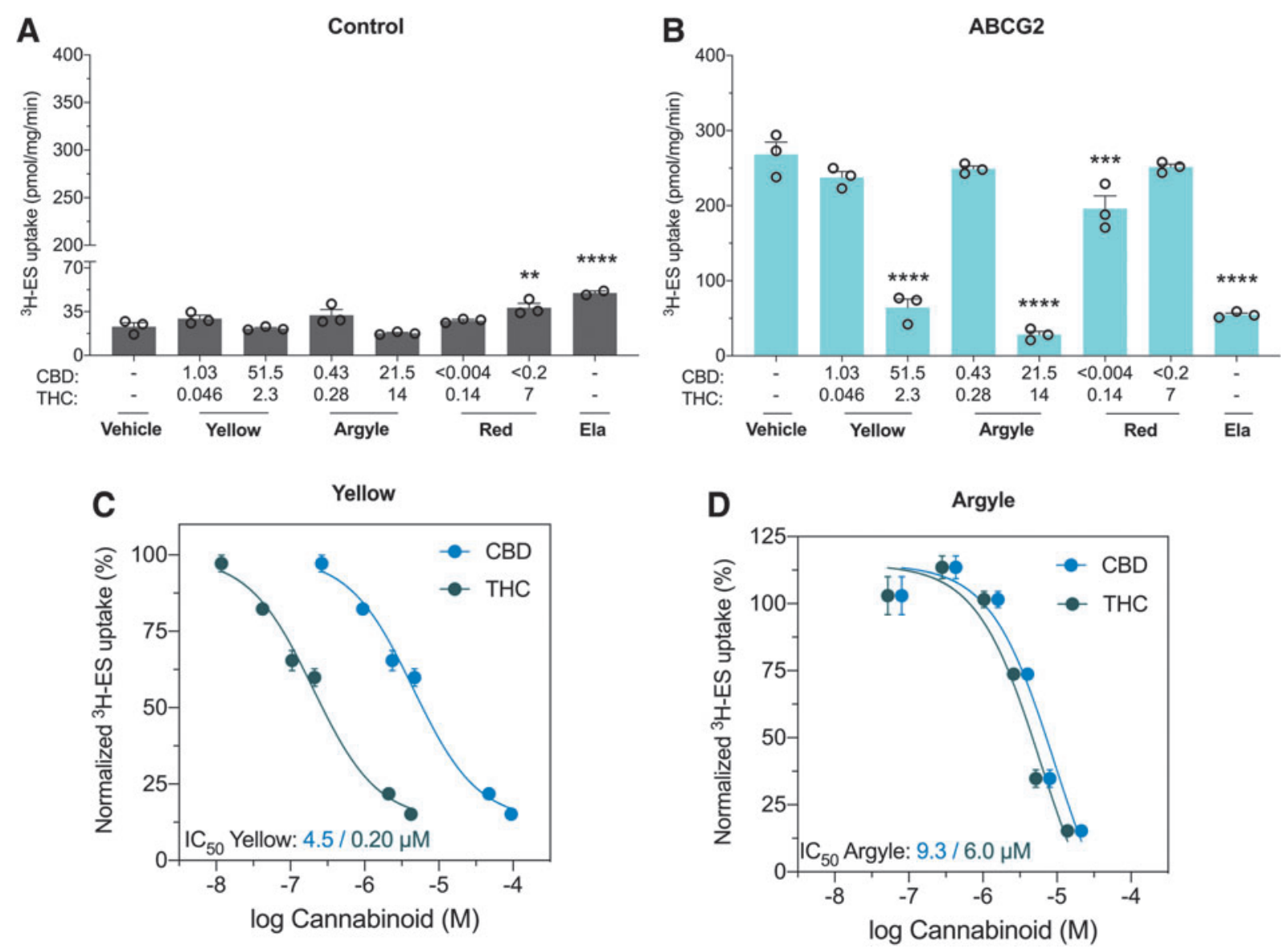

FIG. 2. Cannabis-based products Spectrum Yellow and Tweed Argyle inhibit ABCG2. Inside-out vesicles made from cells expressing human ABCG2, also known as breast cancer resistance protein, were used to screen the inhibitory potential of the three cannabis-based products. Average uptake of ${ }^{3} \mathrm{H}-\mathrm{ES}$ was measured in vesicles from cells (A) transfected with control vector or (B) expressing ABCG2 was quantified by liquid scintillation counting. Spectrum Yellow (Yellow), Tweed Argyle (Argyle), and Spectrum Red (Red) cannabis-based products were applied with final CBD and THC concentrations ( $\mu \mathrm{M})$ depicted (Ela; $5 \mu \mathrm{M})$. High doses of Spectrum Yellow and Tweed Argyle strongly inhibited ABCG2-mediated transport ${ }^{* *} p<0.01$, ${ }^{* * *} p<0.001,{ }^{* * * *} p<0.0001$ compared with vehicle; one-way ANOVA followed by Dunnett's post hoc). Error bars represent SEM, with $n=3$ per group. Concentration-response curves for inhibition of ABCG2 transport of ${ }^{3} \mathrm{H}$-ES by (C) Spectrum Yellow and (D) Tweed Argyle. Uptake of ${ }^{3} \mathrm{H}$-ES was measured in the presence of varying concentrations of Spectrum Yellow and Tweed Argyle, and expressed as a percentage of response to vehicle treatment. Within each of the cannabis-based products, concentrations of THC (green symbols) and CBD (blue symbols) are plotted separately. Data are expressed as mean \pm SEM, with $n=3$ per group. Curves represent fit to a three-parameter log function. Ela, elacridar; ES, estrone 3-sulfate.

appears unlikely that these plasma concentrations could be attained when you consider that $6000 \mathrm{mg}$ Epidiolex ${ }^{\circledR}$ yields an average plasma concentration of $2.5 \mu \mathrm{M}(782 \mathrm{ng} / \mathrm{mL})$ in healthy adults. ${ }^{25}$ In order for Tweed Argyle treatment to achieve an equivalent plasma
CBD concentration, an individual would need to consume $750 \mathrm{~mL}$, which is unlikely given that the $\Delta^{9}$-THC content would be self-limiting due to its psychoactive effects. However, recent data in mice show a pharmacokinetic interaction after $\mathrm{CBD}$ and $\Delta^{9}$-THC 

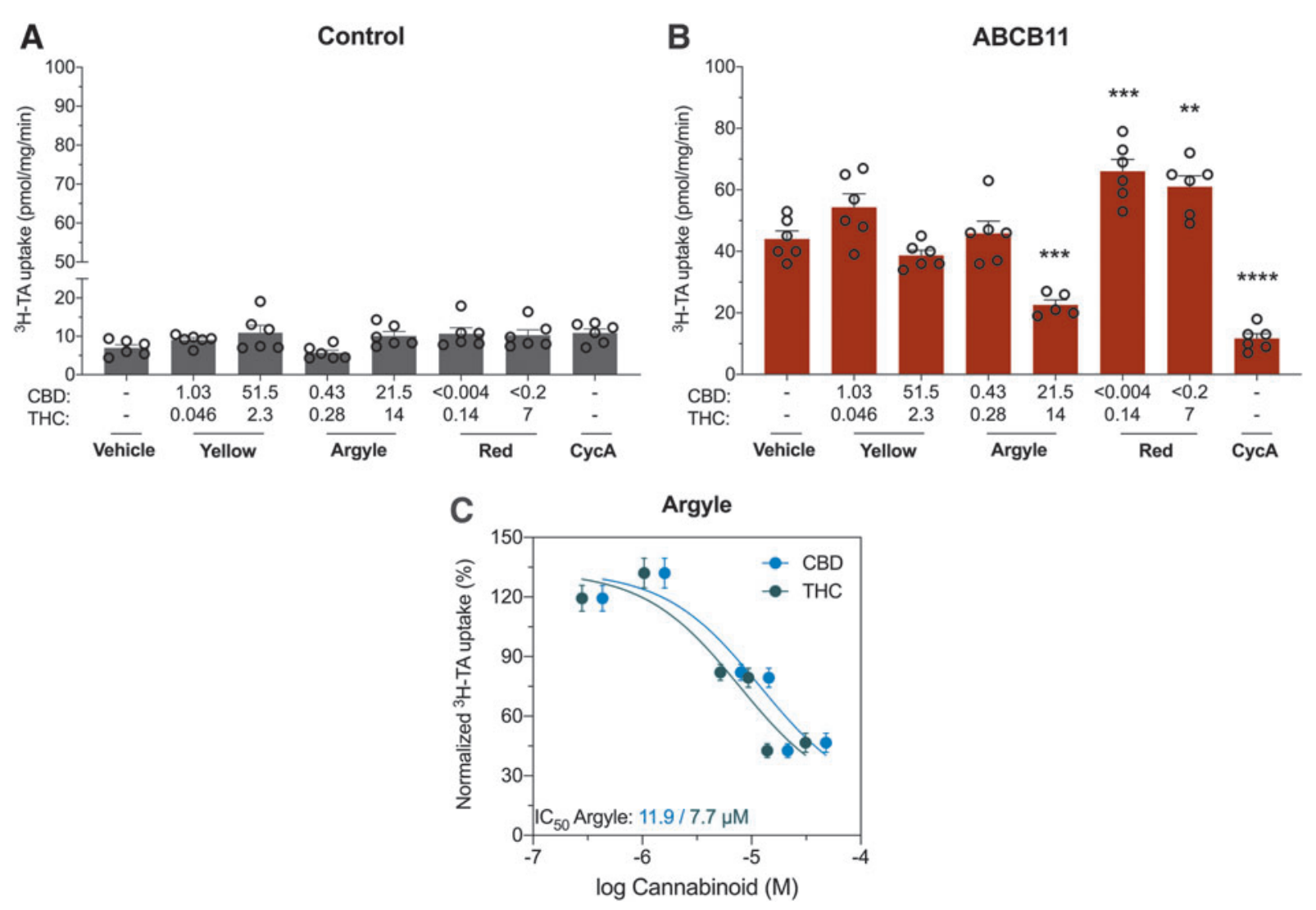

FIG. 3. Tweed Argyle, with balanced $\mathrm{CBD}$ and $\Delta^{9}-\mathrm{THC}$ content, inhibited $\mathrm{ABCB} 11$. Inside-out vesicles made from cells expressing human $A B C B 11$ were used to screen the inhibitory potential of cannabis-based products. Average uptake of ${ }^{3} \mathrm{H}-\mathrm{TA}(1 \mu \mathrm{M})$ was measured in vesicles from cells (A) transfected with control vector or (B) expressing $A B C B 11$ was quantified by liquid scintillation counting. Spectrum Yellow (Yellow), Tweed Argyle (Argyle), and Spectrum Red (Red) cannabis-based products were applied with final CBD and THC concentrations $(\mu \mathrm{M})$ depicted (CycA, $20 \mu \mathrm{M})$. High-dose Tweed Argyle strongly inhibited ABCB11mediated transport $\left({ }^{* *} p<0.005,{ }^{* * *} p<0.0005,{ }^{* * * *} p<0.0001\right.$ compared with vehicle; one-way ANOVA followed by Dunnett's post hoc). Error bars represent SEM, with $n=6$ per group. (C) Concentration-response curves for inhibition of $A B C B 11$ transport of ${ }^{3} \mathrm{H}-\mathrm{TA}$ by Tweed Argyle. Uptake of ${ }^{3} \mathrm{H}$-TA was measured in the presence of varying concentrations of Tweed Argyle and expressed as a percentage of response to vehicle treatment. Within Tweed Argyle, concentrations of THC (green symbols) and CBD (blue symbols) are plotted separately. Data are expressed as mean \pm SEM, with $n=3$ per group. Curves represent fit to a threeparameter log function; TA, taurocholate.

coadministration, which results in significantly increased plasma concentrations of both phytocannabinoids. $^{26}$ In addition, accumulation of CBD and $\Delta^{9}$-THC may occur with chronic administration, which could lead to higher concentrations of both in plasma and tissue. Human pharmacokinetic data after acute and repeated dosing with Tweed Argyle are required to put its inhibitory in vitro effects on $\mathrm{ABCB} 11$ transporter function into perspective.
Cannabis-based products weakly inhibit organic cation transporters

Organic cation transporters (OCTs) are essential for the hepatic and renal elimination of both endogenous and xenobiotic compounds. Approximately $40 \%$ of drugs, including the antidiabetic drug metformin, are organic cations, so inhibition of these transporters could potentially affect drug clearance. ${ }^{27-29}$ All three cannabis-based products weakly inhibited human SLC22A1-mediated 
transport (Fig. 4B). Spectrum Yellow concentration dependently inhibited SLC22A1 transport (13\%, $p=$ 0.0213 and $19 \%, p=0.0008)$. A similar concentrationdependent inhibition was observed with Spectrum Red (19\%, $p=0.0008$ and 25\%, $p<0.0001)$. Tweed Argyle only inhibited SLC22A1-mediated transport at a high concentration $(18 \%, p=0.0017)$. SLC22A2 transporter function was inhibited with the low concentration of Tweed Argyle (22\%, $p=0.009)$ and the high concentration of Spectrum Red (19\%, $p=0.0273$ ) (Fig. 4C).
While the cannabis-based products exhibited some inhibitory potential at OCTs, the modest inhibitions $(<25 \%)$ displayed are unlikely to translate to in vivo DDIs.

\section{Cannabis-based products display mixed} effects at OATs

OATs are important for the renal elimination of low molecular weight anionic molecules. ${ }^{30}$ High concentrations of Spectrum Yellow and Spectrum Red significantly inhibited SLC22A6-mediated transport of ${ }^{3} \mathrm{H}-\mathrm{PAH}$
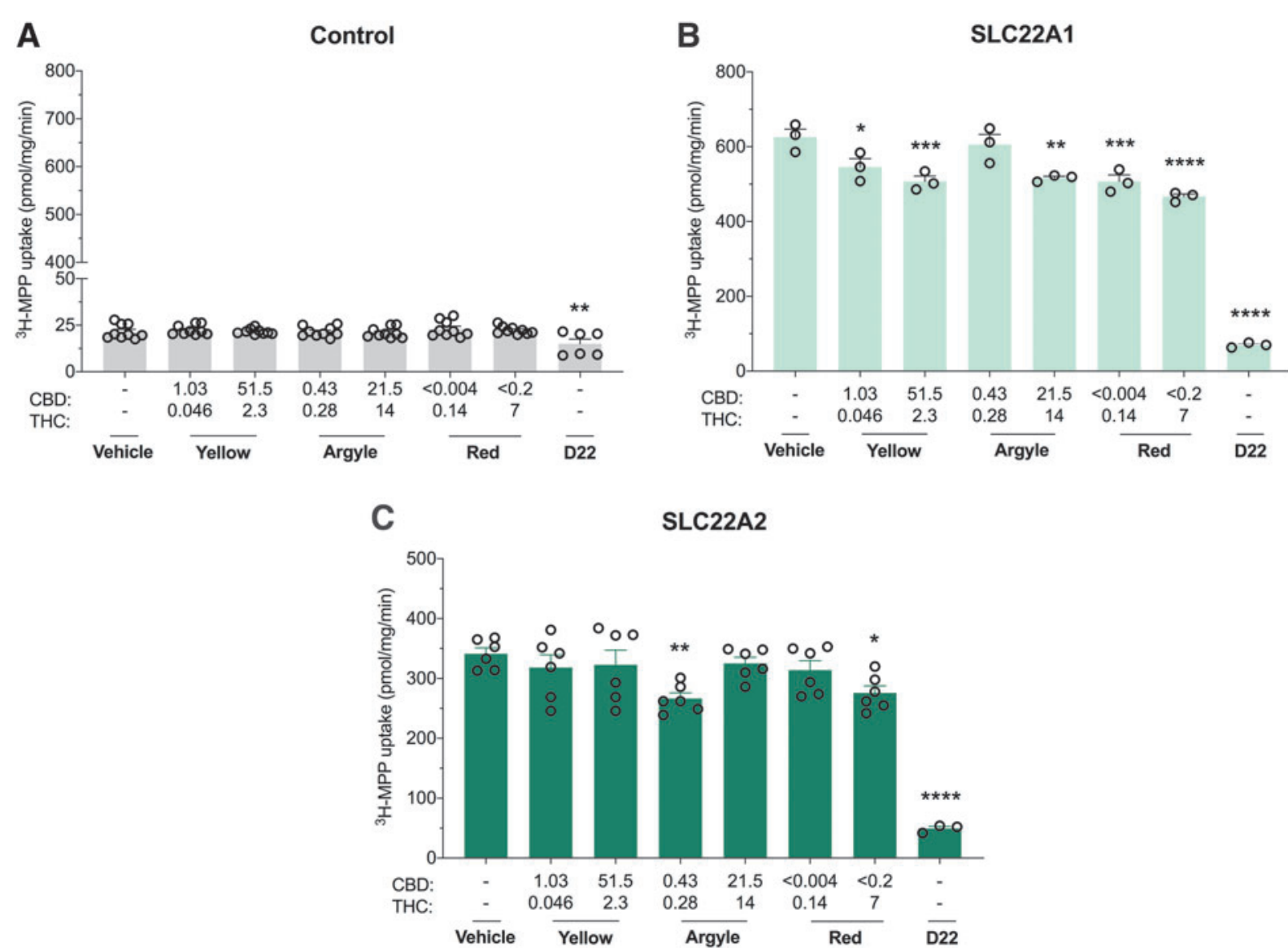

FIG. 4. Cannabis-based products exert weak inhibitory effects on SLC22A1 and SLC22A2. HEK293 cells expressing human organic cation transporters were used to screen the inhibitory potential of cannabis-based products. Average uptake of ${ }^{3} \mathrm{H}-\mathrm{MPP}(10 \mu \mathrm{M})$ into cells (A) transfected with control vector (B) expressing SLC22A1 or (C) expressing SLC22A2 was quantified by liquid scintillation counting. Spectrum Yellow (Yellow), Tweed Argyle (Argyle), and Spectrum Red (Red) cannabis-based products were applied with final CBD and THC concentrations $(\mu \mathrm{M})$ depicted (D22, $50 \mu \mathrm{M})$. Weak inhibition of SLC22A1 was observed with Spectrum Yellow, Tweed Argyle, and Spectrum Red $\left({ }^{*} p<0.05,{ }^{* *} p<0.005,{ }^{* *} p<0.001,{ }^{* * * *} p<0.0001\right.$ compared with vehicle; one-way ANOVA followed by Dunnett's post hoc). Error bars represent SEM, with $n=3$ per group. Lowdose Tweed Argyle and high-dose Spectrum Red weakly inhibited SLC22A2 transport ${ }^{*} p<0.05,{ }^{* *} p<0.01$, ${ }^{* * * *} p<0.0001$ compared with vehicle; one-way ANOVA followed by Dunnett's post hoc). Error bars represent SEM, with $n=3-6$ per group. MPP, 1-methyl-4-phenylpyridinium iodide; D22, Decynium-22. 
(34\%, $p<0.0001$ and $27 \%, p=0.0008$, respectively) (Fig. 5B). High concentrations of all cannabis-based products significantly inhibited SLC22A8-mediated transport of ${ }^{3} \mathrm{H}-\mathrm{ES}$ (Spectrum Yellow, 45\%; Tweed Argyle, 19\%; Spectrum Red, 31\%; $p<0.0001$ ) (Fig. 5D).

Since OATs are essential for elimination of anionic molecules including uremic toxins, gut viral metabo- lites, and antimicrobials, inhibiting this family of transporters could lead to serious DDIs. ${ }^{30,31}$ High concentrations of the cannabis-based products only modestly inhibited SLC22A6 and SLC22A8 transporter function in comparison with probenecid, so the relevance of these interactions to an in vivo DDI is doubtful but could be explored further.
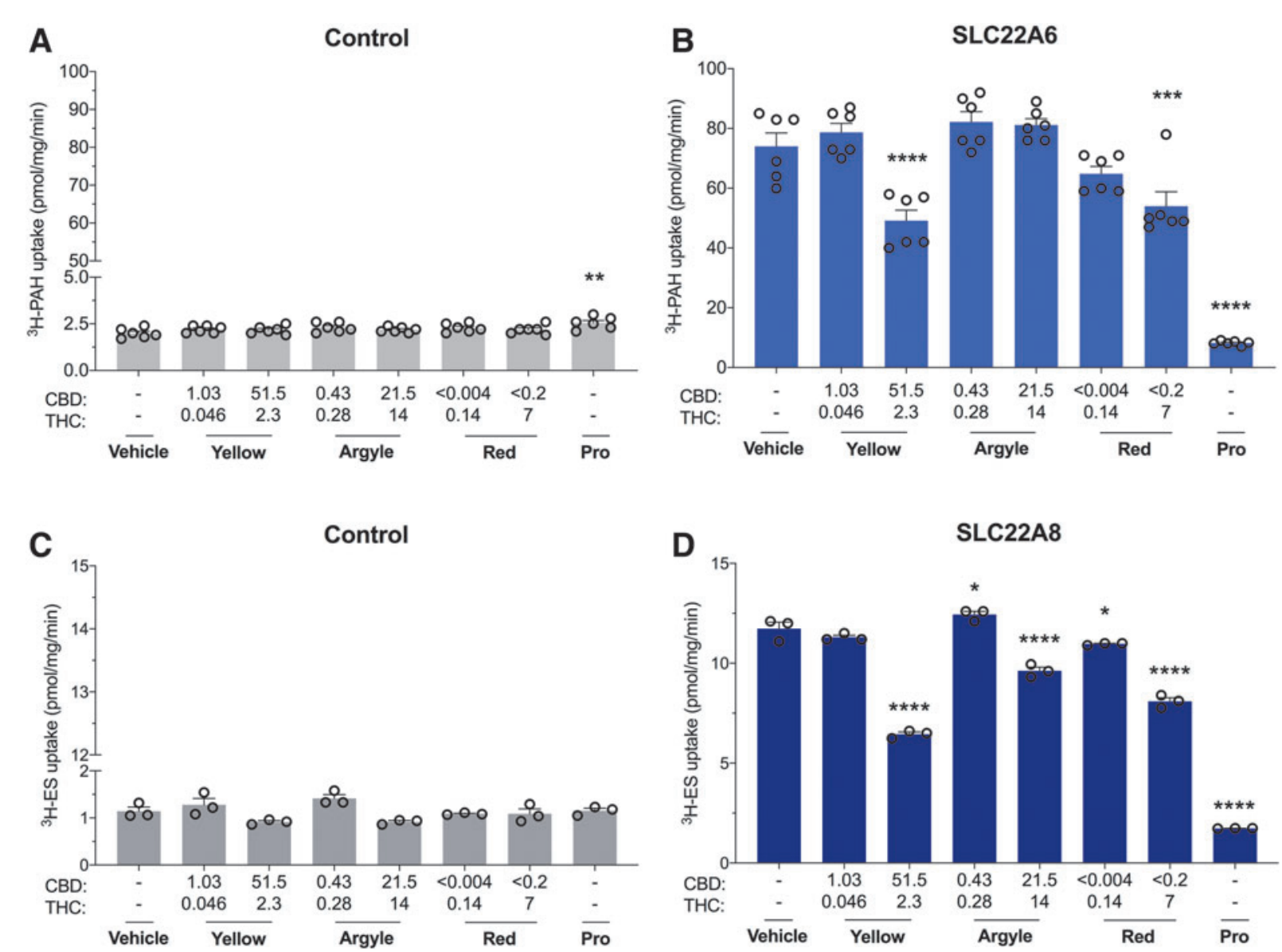

FIG. 5. High concentrations of Spectrum Yellow and Spectrum Red cannabis-based products modestly inhibit SLC22A6 but not SLC22A8. Cells expressing human organic anion transporters were used to screen the inhibitory potential of three cannabis-based products. Average uptake of ${ }^{3} \mathrm{H}-\mathrm{PAH}(10 \mu \mathrm{M})$ into cells (A) transfected with control vector or (B) expressing SLC22A6 was quantified by liquid scintillation counting. Spectrum Yellow (Yellow), Tweed Argyle (Argyle), and Spectrum Red (Red) cannabis-based products were applied with final CBD and THC concentrations $(\mu \mathrm{M})$ depicted (Pro, $100 \mu \mathrm{M})$. High doses of both Spectrum Yellow and Spectrum Red weakly inhibited SLC22A6 transport ${ }^{* * *} p<0.001$, ${ }^{* * * *} p<0.0001$ compared with vehicle; one-way ANOVA followed by Dunnett's post hoc). Error bars represent SEM, with $n=6$ per group. Average uptake of ${ }^{3} \mathrm{H}-\mathrm{ES}(1 \mu \mathrm{M})$ into cells (C) transfected with control vector or (D) expressing SLC22A8 was quantified by liquid scintillation counting (Pro, $100 \mu \mathrm{M}$ ). High concentrations of cannabis oils modestly inhibited SLC22A8 transport $\left({ }^{*} p<0.05,{ }^{* * * *} p<0.0001\right.$ compared with vehicle; oneway ANOVA followed by Dunnett's post hoc). Error bars represent SEM, with $n=3$ per group. Pro, probenecid; $\mathrm{PAH}, p$-aminohippuric acid. 
Cannabis-based products inhibit organic anion transporting polypeptides

Organic anion transporting polypeptides (OATPs) mediate the transport of large amphipathic organic anions across biological membranes. High concentrations of
Spectrum Yellow and Tweed Argyle inhibited SLCO1B1mediated transport of ${ }^{3} \mathrm{H}-\mathrm{ES}$ (42\%, $p<0.0001$ and $22 \%, p<0.0001$, respectively) and SLCO1B3-mediated uptake of ${ }^{3} \mathrm{H}$-sulfobromophthalein (37\%, $p<0.0001$ and $22 \%, p<0.0001$ ) (Fig. 6B, D). Interestingly,
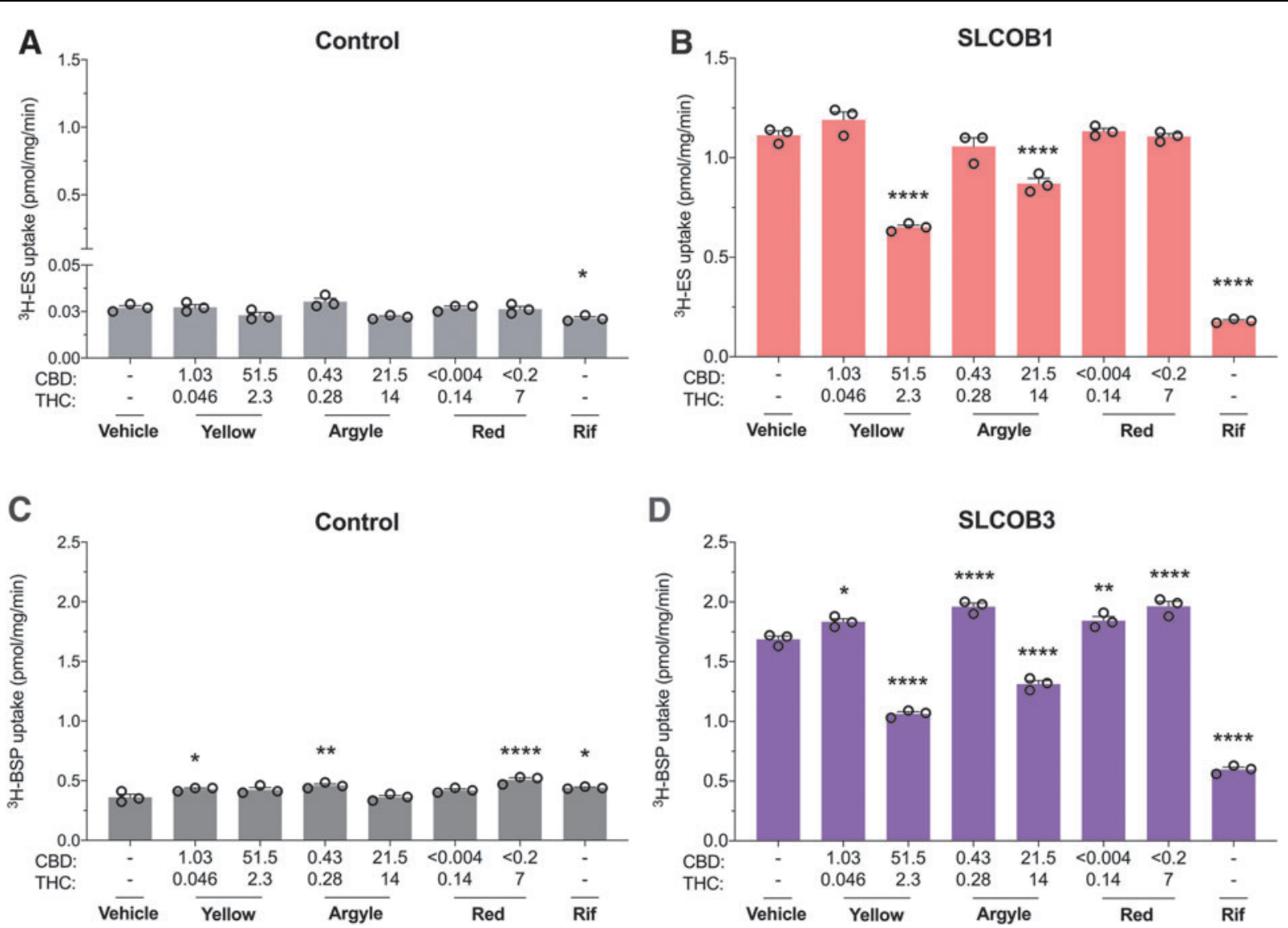

FIG. 6. High concentrations of Spectrum Yellow and Tweed Argyle cannabis-based products modestly inhibit SLCO1B1 and SLCO1B3. Cells expressing human organic anion transporting polypeptides were used to screen the inhibitory potential of three cannabis-based products. Average uptake of ${ }^{3} \mathrm{H}-\mathrm{ES}(0.03 \mu \mathrm{M})$ into cells (A) transfected with control vector or (B) expressing SLCO1B1 was quantified by liquid scintillation counting. Spectrum Yellow (Yellow), Tweed Argyle (Argyle), and Spectrum Red (Red) cannabis-based products were applied with final CBD and THC concentrations $(\mu \mathrm{M})$ depicted (Rif, $50 \mu \mathrm{M}$ ). High doses of both Spectrum Yellow and Tweed Argyle inhibited SLCO1B1 transport $\left({ }^{*} p<0.05\right.$, ${ }^{* * *} p<0.0001$ compared with vehicle; one-way ANOVA followed by Dunnett's post hoc). Error bars represent SEM, with $n=3$ per group. Average uptake of ${ }^{3} \mathrm{H}-\mathrm{BSP}(0.05 \mu \mathrm{M})$ into cells (C) transfected with control vector or (D) expressing SLCO1B3 was quantified by liquid scintillation counting (Rif, $5 \mu \mathrm{M}$ ). In control cells, uptake of ${ }^{3} \mathrm{H}$-BSP increased in the presence of cannabis oils $\left({ }^{*} p<0.05,{ }^{* *} p<0.005,{ }^{* * *} p<0.0001\right.$ compared with vehicle; one-way ANOVA followed by Dunnett's post hoc). Error bars represent SEM, with $n=3$ per group. Uptake of ${ }^{3} \mathrm{H}-\mathrm{BSP}$ was increased in SLCO1B3-expressing cells after treatment with Spectrum Red. Low doses of both Spectrum Yellow and Tweed Argyle also increased ${ }^{3} \mathrm{H}-\mathrm{BSP}$ uptake. SLCO1B3 transport was inhibited by high doses of Spectrum Yellow and Tweed Argyle $\left({ }^{*} p<0.05,{ }^{* *} p<0.01,{ }^{* * * *} p<0.0001\right.$ compared with vehicle; one-way ANOVA followed by Dunnett's post hoc). Error bars represent SEM, with $n=3$ per group. Rif, rifampicin; BSP, sulfobromophthalein. 
low concentrations of Spectrum Yellow and Tweed Argyle appeared to slightly increase uptake of ${ }^{3} \mathrm{H}$-sulfobromophthalein $(p=0.0161$ and $p<0.0001$, respectively); however, a similar increase was also observed in control cells suggesting a nonspecific effect (Fig. 6C).
OATPs are involved in elimination of endogenous and xenobiotic substrates, including bilirubin, thyroid hormones, antibiotics, anticancer drugs, and cholesterol lowering statins, so any impact on transporter function could translate into a meaningful DDI. ${ }^{32,33}$ High concentrations of Spectrum Yellow and Tweed Argyle
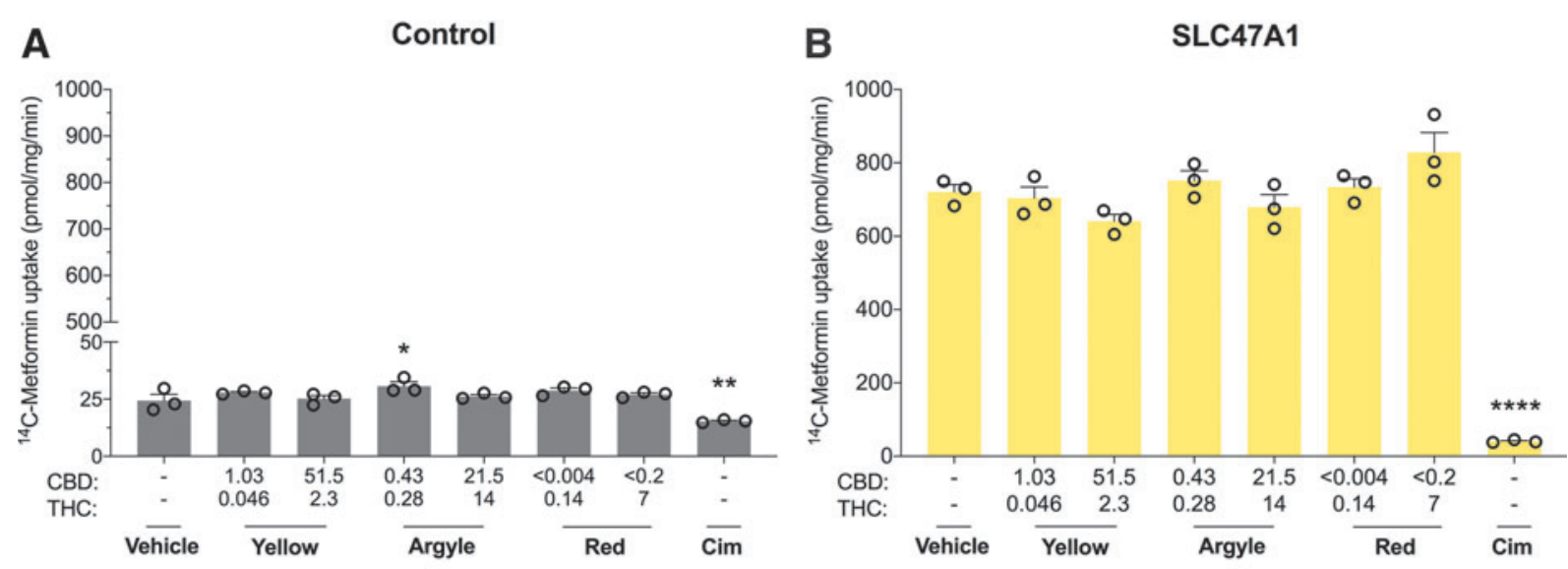

C

Control

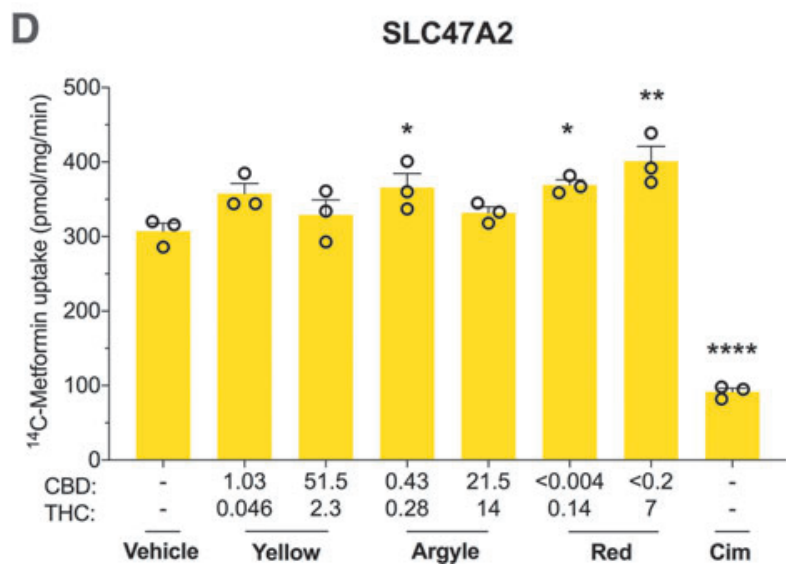

FIG. 7. The three cannabis-based products did not inhibit SLC47A1 or SLC47A2 transporters. Cells expressing human multidrug and toxin extrusion transporters were used to screen the inhibitory potential of three cannabis-based products. Average uptake of ${ }^{14} \mathrm{C}$-Metformin $(20 \mu \mathrm{M})$ into cells (A) transfected with control vector or (B) expressing SLC47A1 was quantified by liquid scintillation counting. Spectrum Yellow (Yellow), Tweed Argyle (Argyle), and Spectrum Red (Red) cannabis-based products were applied with final CBD and THC concentrations $(\mu \mathrm{M})$ depicted $(\mathrm{Cim}, 50 \mu \mathrm{M})$. None of the cannabis-based products affected SLC47A1 transport. Error bars represent SEM, with $n=3$ per group. Average uptake of ${ }^{14} \mathrm{C}$-Metformin $(90 \mu \mathrm{M})$ into cells (C) transfected with control vector or (D) expressing SLC47A2 was quantified by liquid scintillation counting $(\mathrm{Cim}, 50 \mu \mathrm{M})$. In control cells, uptake of ${ }^{14} \mathrm{C}$-Metformin increased in the presence of the cannabis-based products $\left({ }^{*} p<0.05,{ }^{* * *} p<0.001,{ }^{* * * *} p<0.0001\right.$ compared with vehicle; one-way ANOVA followed by Dunnett's post hoc). Error bars represent SEM, with $n=3$ per group. Spectrum Red and lowdose Tweed Argyle slightly increased uptake of ${ }^{14} \mathrm{C}$-Metformin $\left({ }^{*} p<0.05,{ }^{* *} p<0.005,{ }^{* * * *} p<0.0001\right.$ compared with vehicle; one-way ANOVA followed by Dunnett's post hoc). Error bars represent SEM, with $n=3$ per group. Cim, cimetidine. 
only modestly inhibited OATP transporter function in comparison with rifampicin.

\section{Cannabis-based products did not inhibit} multidrug and toxin extrusion transporters

Multidrug and toxin extrusion transporters are important for renal elimination of various endogenous and xenobiotic substrates. None of the cannabis-based products affected SLC47A1 transport (Fig. 7B). Tweed Argyle slightly enhanced SLC47A2 transport $(p=0.0455)$ (Fig. 4D). Spectrum Red concentration dependently increased ${ }^{14} \mathrm{C}$-metformin uptake in SLC47A2-expressing cells ( $p=0.0327$ and $p=0.0012$ ); however, since this increase was also observed in control cells it suggests an effect nonspecific to SLC47A2 (Fig. 7C, D). Overall, none of the three cannabisbased products inhibited SLC47A1 or SLC47A2 transport, so an in vivo DDI would not be predicted.

\section{Conclusion}

The three cannabis-based products did not inhibit ABCC2, ABCB1, SLC47A1, SLC47A2, or SLC22A8 transporters, so in vivo DDIs mediated by these transporters are remote. Intriguingly, cannabis-based products increased transporter function in $\mathrm{ABCB} 1$-expressing cells, but this was likely nonspecific to ABCB1. Novel findings were that these cannabis-based products inhibited ABCB11, SLC22A6, SLC22A1, SLC22A2, SLCO1B1, and SLCO1B3, and future studies could further explore these interactions. However, Spectrum Yellow and Tweed Argyle inhibited ABCG2 transporter function at concentrations that may be clinically relevant; therefore, future in vivo DDI studies for ABCG2 inhibition might be justified.

\section{Acknowledgments}

The authors gratefully acknowledge Barry and Joy Lambert for their continued support of the Lambert Initiative for Cannabinoid Therapeutics. They thank Declan Morgan and Professor Dr. Yohannes Hagos for their technical assistance.

\section{Author Disclosure Statement}

J.C.A. is Deputy Academic Director of the Lambert Initiative for Cannabinoid Therapeutics. He has served as an expert witness in various medicolegal cases involving cannabis, and served as a temporary advisor to the World Health Organization (WHO) on their review of cannabis and the cannabinoids. His research is funded by the Australian National Health and
Medical Research Council, (APP1161571) and the Lambert Initiative for Cannabinoid Therapeutics. J.C.A and L.L.A are inventors on several patents involving cannabinoid therapeutics. L.M., M.H.L., I.M., and M.O.B.-M. are employees of Canopy Growth Corporation. M.H.L. was a prior employee of Greenwich Biosciences. M.O.B.-M. also serves on the Board of Directors for AusCann Group Holdings Limited, was a prior employee of Zynerba Pharmaceuticals, and has received consulting fees from Tilray Inc.

\section{Funding Information}

This research was funded by Canopy Growth Corporation.

\section{Supplementary Material}

Supplementary Table S1

\section{References}

1. Stout SM, Cimino NM. Exogenous cannabinoids as substrates, inhibitors, and inducers of human drug metabolizing enzymes: a systematic review. Drug Metab Rev. 2014;46:86-95.

2. Geffrey AL, Pollack SF, Bruno PL, et al. Drug-drug interaction between clobazam and cannabidiol in children with refractory epilepsy. Epilepsia. 2015;56:1246-1251.

3. Gaston TE, Bebin EM, Cutter GR, et al. Interactions between cannabidiol and commonly used antiepileptic drugs. Epilepsia. 2017;58:1586-1592.

4. ter Beek J, Guskov A, Slotboom DJ. Structural diversity of $A B C$ transporters. J Gen Physiol. 2014;143:419-435.

5. Glavinas $H$, Krajcsi $P$, Cserepes J, et al. The role of $A B C$ transporters in drug resistance, metabolism and toxicity. Curr Drug Deliv. 2004;1:27-42.

6. Zhu HJ, Wang JS, Markowitz JS, et al. Characterization of P-glycoprotein inhibition by major cannabinoids from marijuana. J Pharmacol Exp Ther. 2006;317:850-857.

7. Tournier N, Chevillard L, Megarbane B, et al. Interaction of drugs of abuse and maintenance treatments with human P-glycoprotein (ABCB1) and breast cancer resistance protein (ABCG2). Int J Neuropsychopharmacol. 2010;13:905-915.

8. Feinshtein V, Erez O, Ben-Zvi Z, et al. Cannabidiol enhances xenobiotic permeability through the human placental barrier by direct inhibition of breast cancer resistance protein: an ex vivo study. J Obstet Gynecol. 2013; 209:573.e1-573.e15.

9. Holland $M L$, Lau $D$, Allen JD, et al. The multidrug transporter ABCG2 (BCRP) is inhibited by plant-derived cannabinoids. Br J Pharmacol. 2007; 152:815-824.

10. Holland ML, Allen JD, Arnold JC. Interaction of plant cannabinoids with the multidrug transporter ABCC1 (MRP1). Eur J Pharmacol. 2008;591:128131.

11. Carrier EJ, Auchampach JA, Hillard CJ. Inhibition of an equilibrative nucleoside transporter by cannabidiol: a mechanism of cannabinoid immunosuppression. Proc Natl Acad Sci U S A. 2006;103: 7895-7900.

12. Brenneiser R, Egli A, Elsohly MA, et al. The effect of orally and rectally administered delta 9-tetrahydrocannabinol on spasticity: a pilot study with 2 patients. Int J Clin Pharmacol Ther. 1996;34:446-452.

13. Karlsson JE, Heddle C, Rozkov A, et al. High-activity p-glycoprotein, multidrug resistance protein 2 , and breast cancer resistance protein membrane vesicles prepared from transiently transfected human embryonic kidney 293-epstein-barr virus nuclear antigen cells. Drug Metab Dispos. 2010;38:705-714.

14. Chen Z, Shi T, Zhang L, et al. Mammalian drug efflux transporters of the ATP binding cassette $(A B C)$ family in multidrug resistance: a review of the past decade. Cancer Lett. 2016;370:153-164. 
15. Arnold JC, Hone P, Holland ML, et al. CB2 and TRPV1 receptors mediate cannabinoid actions on MDR1 expression in multidrug resistant cells. Pharmacol Rep. 2012;64:751-757.

16. Brzozowska NI, de Tonnerre EJ, Li KM, et al. The differential binding of antipsychotic drugs to the $A B C$ transporter p-glycoprotein predicts cannabinoid-antipsychotic drug interactions. Neuropsychopharmacology. 2017;42:2222-2231.

17. Van der Sandt ICJ, Blom-Roosemalen MCM, de Boer AG, et al. Specificity of doxorubicin versus rhodamine-123 in assessing P-glycoprotein functionality in the LLC-PK1, LLC-PK1:MDR1 and Caco-2 cell lines. Eur J Pharm Sci. 2000;11:207-214.

18. Srivalli KMR, Lakshmi PK. Overview of P-glycoprotein inhibitors: a rational outlook. Braz J Pharm Sci. 2012;48:353-367.

19. Molnár J, Szabó D, Puszatai R, et al. Membrane associated antitumor effects of crocine-, ginsenoside- and cannabinoid derivates. Anticancer Res. 2000;20:861-867.

20. Holland ML, Panetta JA, Hoskins JM, et al. The effects of cannabinoids on P-glycoprotein transport and expression in multidrug resistant cells. Biochem Pharmacol. 2006;71:1146-1154.

21. Schinkel $A H$, Jonker JW. Mammalian drug efflux transporters of the ATP binding cassette (ABC) family: an overview. Adv Drug Deliv Rev. 2003;55: 3-29.

22. Lee $C A, O^{\prime} C o n n o r ~ M A, ~ R i t c h i e ~ T K$, et al. Breast cancer resistance protein (ABCG2) in clinical pharmacokinetics and drug interactions: practical recommendations for clinical victim and perpetrator drug-drug interaction study design. Drug Metab Dispos. 2015;43:490-509.

23. Jetter A, Kullak-Ublick GA. Drugs and hepatic transporters: a review. Pharmacol Res. 2020;154:104234.

24. Kenna JG, Taskar KS, Battista C, et al. Can bile salt export pump inhibition testing in drug discovery and development reduce liver injury risk? An international transporter consortium perspective. Clin Pharmacol Ther. 2018;104:916-932.

25. Taylor L, Gidal B, Blakey G, et al. A phase I, randomized, double-blind, placebo-controlled, single ascending dose, multiple dose, and food effect trial of the safety, tolerability and pharmacokinetics of highly purified cannabidiol in healthy subjects. CNS Drugs. 2018;32: 1053-1067.

26. Anderson LL, Low IK, McGregor IS, et al. Interactions between cannabidiol and $\Delta 9$-tetrahydrocannabinol in modulating seizure susceptibility and survival in a mouse model of Dravet syndrome. Br J Pharmacol. 2020; 177:4261-4274.

27. Koepsell H. Organic cation transporters in health and disease. Pharmacol Rev. 2020;72:253-319.

28. Kimura N, Masuda S, Tanihara Y, et al. Metformin is a superior substrate for renal organic cation transporter OCT2 rather than hepatic OCT1. Drug Metab Pharmacokinet. 2005;20:379-386.

29. Hacker K, Maas R, Kornhuber J, et al. Substrate-dependent inhibition of the human organic cation transporter OCT2: a comparison of metformin with experimental substrates. PLoS One. 2015;10:e0136451.

30. VanWert AL, Gionfriddo MR, Sweet DH. Organic anion transporters: discovery, pharmacology, regulation and roles in pathophysiology. Biopharm Drug Dispos. 2010;31:1-71.
31. Nignam SK, Bush KT, Martovetsky G, et al. The organic anion transporter (OAT) family: a systems biology perspective. Physiol Rev. 2015;95: 83-123.

32. Stieger B, Hagenbuch B. Organic anion transporting polypeptides. Curr Top Membr. 2014;73:205-232.

33. Shitara Y, Maeda K, Ikejiri K, et al. Clinical significance of organic anion transporting polypeptides (OATPs) in drug disposition: their roles in hepatic clearance and intestinal absorption. Biopharm Drug Dispos. 2013; 34:45-78.

Cite this article as: Anderson LL, Etchart MG, MacNair L, Hunter Land M, Mosesova IA, Bonn-Miller MO, Arnold JC (2022) In vitro screening of three commercial cannabis-based products on ATP-binding cassette and solute-carrier transporter function, Cannabis and Cannabinoid Research 7:3, 304-317, DOI: 10.1089/can.2020.0053.

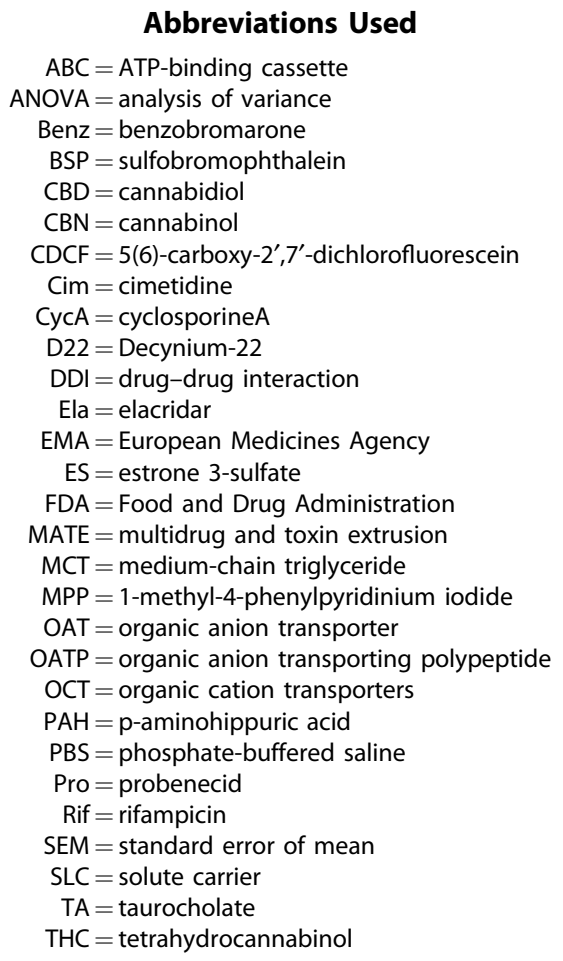

Abbreviations Used

$\mathrm{ABC}=\mathrm{ATP}$-binding cassette

ANOVA $=$ analysis of variance

Benz $=$ benzobromarone

$\mathrm{BSP}=$ sulfobromophthalein

$\mathrm{CBD}=$ cannabidiol

$\mathrm{CBN}=$ cannabinol

$\mathrm{CDCF}=5(6)$-carboxy-2', $7^{\prime}$-dichlorofluorescein

Cim $=$ cimetidine

$\mathrm{CycA}=$ cyclosporine $\mathrm{A}$

D22 = Decynium-22

$\mathrm{DDI}=$ drug-drug interaction

Ela $=$ elacridar

$\mathrm{EMA}=$ European Medicines Agency

$E S=$ estrone 3-sulfate

$\mathrm{FDA}=$ Food and Drug Administration

$M A T E=$ multidrug and toxin extrusion

$\mathrm{MCT}=$ medium-chain triglyceride

MPP =1-methyl-4-phenylpyridinium iodide

OAT $=$ organic anion transporter

OATP $=$ organic anion transporting polypeptide

$\mathrm{OCT}=$ organic cation transporters

$\mathrm{PAH}=\mathrm{p}$-aminohippuric acid

$\mathrm{PBS}=$ phosphate-buffered saline

Pro $=$ probenecid

Rif $=$ rifampicin

SEM $=$ standard error of mean

$\mathrm{SLC}=$ solute carrier

$\mathrm{TA}=$ taurocholate

$\mathrm{THC}=$ tetrahydrocannabinol 